Französische Literatur in Einzeldarstellungen 
Peter Brockmeier/Hermann H. Wetzel (Hrsg.)

\section{Französische Literatur in Einzeldarstellungen}

Band 1

Von Rabelais bis Diderot

J. B. Metzlersche Verlagsbuchhandlung Stuttgart 
Französische Literatur in Einzeldarstellungen /

Peter Brockmeier ; Hermann H. Wetzel (Hrsg.). -

Stuttgart : Metzler

NE: Brockmeier, Peter 〈Hrsg. $\rangle$

Bd. 1. Von Rabelais bis Diderot. - 1981.

ISBN 978-3-476-00473-4

ISBN 978-3-476-00473-4

ISBN 978-3-476-03150-1 (eBook)

DOI 10.1007/978-3-476-03150-1

(C) 1981 Springer-Verlag GmbH Deutschland

Ursprünglich erschienen bei J.B. Metzlersche Verlagsbuchhandlung und Carl Ernst Poeschel Verlag GmbH in Stuttgart 1981 


\title{
Inhalt
}

\author{
Vorwort 9
}

Frank-Rutger Hausmann: Rabelais und das Aufkommen des Absolutismus.

Religion, Staat und Hauswesen

in den fünf Büchern Gargantua et Pantagruel 13

1. Rabelais und seine Zeit 13; 2. Rabelais' Leben und Werk 16; 3. Kurzer Forschungsbericht 17; 4. Voraussetzungen einer Rabelais-Lektüre: "Die Abtei von Thelema " (Gargantua, Kap. 49-56); die Allegorese 18; 5. Lesemodell für eine mehrschichtige Rabelais-Lektüre 25; 6. "Die Glocken von Notre-Dame " ( Gargantua, Kap. 16-19): Das Volk von Paris, die Theologen der Sorbonne, die Parlamentsjuristen 28; 7. "Der pikrocholinische Krieg" (Gargantua, Kap. 23-48): Krieg und Herrschaftsausübung 33; 8. "Das Lob auf Schuldner und Borger " (Tiers Livre, Kap. 2-5): Beginnender Kapitalismus und Rittertum 38; 9. "Der Würstekrieg" (Quart Livre, Kap. 35-42): Das Zeitalter der Glaubenskriege 45; 10. "Die gefrorenen Worte" (Quart Livre, Kap. 55-56): Das Zeitalter der Entdeckungen 50; 11. Schlußbetrachtung 54; Anmerkungen 56; Verzeichnis der Abkürzungen 75.

Peter Bürger: Zum Funktionswandel der dramatischen Literatur in der Epoche des entstehenden Absolutismus 77

1. Vorbemerkung: Zum Problem literarischer Evolution 77; 2. "La Querelle du Cid«. Zur Institutionalisierung der "doctrine classique» 79; 3. Corneilles Cid und das Material der Tragikomödie 91; 4. Materialien 102; Anmerkungen 111.

\section{Volker Kapp: Die Idealisierung der höfischen Welt im klassischen Drama 115}

1. Einleitung 115; 2. Der Illusionscharakter der klassischen Bühne und die Idealisierung der höfischen Welt durch das klassische Drama 117; 3. Die Deutung der "vraisemblance " bei d'Aubignac durch die wechselseitige Beziehung zwischen Rolle in der Gesellschaft und Rolle auf der Bühne und die Besonderheiten der aristokratischen Lebensform im Frankreich des 17. Jahrhunderts 125 ; 4. Die Forderung nach Respektierung der "bienséance" im Theater und der mit der Festigung des Absolutismus verbundene Wandel der aristokratischen Zielvorstellungen 131; 5. Das Interesse des Absolutismus an der Verfeinerung der Sitten und die Entwicklung von Corneilles Theater bis zum Ende der Fronde 143; 6. Die Verankerung der höfischen Welt in der Natur des Menschen durch Molière und die Identitätsproblematik bei Racine 157; Anmerkungen 173. 
1. Begriff und Wort "Moralistik« 177; 2. Moralistische Autoren 180; 3. Kontext der Erziehungsbücher 183; 4. Geselligkeit und Öffentlichkeit 186; 5. Antike Tradition und religiöser Kontext 188; 6. Gesellschaftlicher Kontext 189; 7. Moralistisches Normenangebot 190; 7.1. An gesellschaftlicher Praxis orientierte Normen 191; 7.2. Gegen gesellschaftliche Praxis gerichtete Normen 193； 8. "Honnête homme " als Norm 197; 9. "Honnête homme" als Bildungsnorm 200; 10. Bedeutung der Frau 202; 11. "Plaire" als Verhaltensnorm 203; 12. Normierung des Umgangs mit Leidenschaften und "médiocrité " als Norm 204; 13. Begriff der Norm 208; 14. Abstraktionsgrad von Normen und Normenanwendung 209; 15. Aphorismus und Systemlosigkeit 210; 16. Aphoristische Formen 211; 17. Beliebtheit moralistischer Normierungen 214; Anmerkungen 215.

Günter Berger: Oppositionelle Literatur zu Anfang des 17. Jahrhunderts

1. Einleitung $225 ;$ 1.1. Staat und Gesellschaft Frankreichs von 1600-1630 225; 1.2 . Überblick über die Literatur der Jahre 1600-1630 227; 1.2.1. Lyrik 227; 1.2.2. Theater 228; 1.2.3. Erzählende fiktionale Prosa 228; 2. Oppositionelle Literatur 229; 2.1. Theóphile de Viau (1590-1626) 229; 2.2. Sorels Francion 242; 3. Oppositionelle Literatur und libertinistischer Hofadel 251; Anmerkungen 254; Bibliographie 259.

\section{Thomas Schleich: Literatur der Aufklärung und gesellschaftlicher Wandel- Ursachen, Erscheinungsformen und Nachwirkungen politischer Reflexion im 18. Jahrhundert in Frankreich 261}

0. Aufklärung als sozialgeschichtlicher Prozeß 261; 1. Der prekäre Fortschritt: Grundstrukturen und Wandlungsprozesse in Staat, Gesellschaft und Wirtschaft 265; 2. Das Spektrum aufklärerischer Politik - Erscheinungsformen politischen Denkens 277; 2.1. Trägerschichten und Medien der Verbreitung 277; 2.2. Literarische Modelle und Ausformungen 285 ; 3. Etappen in der Entwicklung des Selbstverständnisses der Aufklärer: Exemplarische Textinterpretationen 288; 3.1. Die Lettres persanes von Montesquieu (1721) 289; 3.2. L'Ecumoire von Crébillon fils (1734) 291; 3.3. De l'esprit von Helvétius (1758) 293; 3.4. L'homme aux quarante écus von Voltaire (1768) 296; 3.5. L'an 2440 von Mercier (1771) 298; 3.6. Essai sur les règnes de Claude et de Néron von Didérot (1778/1782) 300; 4. Zu den Nachwirkungen - die verschiedenen Gesichter der Aufklärung 302; Anmerkungen 311.

\section{Peter Brockmeier: Die Kritik der Vorurteile} in der französischen Literatur des 18. Jahrhunderts

1. Der Kampf gegen die Vorurteile 321；1.1. Kritik und Verblendung 321；1.2. Grundvorstellungen der Aufklärung: Vorurteil, Philosophie, Mensch 322; 1.3. Themen und Ziele der Vorurteilskritik (Fontenelle, Bayle, Meslier, d'Holbach, Voltaire) 327; 1.4. Notwendige Vorurteile und die Vorbereitung des Wahlzensus 336; 2. Die Kritik des Despotismus und der Religion, des Standesdünkels und der Metaphysik bei Montesquieu, Marivaux und Voltaire 341; 2.1. Ein Meisterwerk der Persiflage: Lettres persanes (1721) 341; 2.2. Standesgrenzen und Humanität: Marivaux' L'Ile des esclaves (1725) 346; 2.3. Soziale Integration 
eines Aufsteigers: Le Paysan parvenu (1734) 350; 2.4. Ironie und Skepsis des unvoreingenommenen Philosophen: Le monde comme il va. Vision de Babouc (1748), Candide ou l'Optimisme (1759) 354; 3. Die Kritik an Fortschritt und Zivilisation im Werk Jean-Jacques Rousseaus $362 ; 3.1$. Der allmächtige Reiche und der machtlose Arme 362; 3.2. Der natürliche Mensch und die verdorbene Gesellschaft 363; 3.3. Kritik des Standesdünkels in Julie ou la Nouvelle Héloïse (1761) 368; 4. Kritik der Vorurteile vom Standpunkt des Libertins: Zum Werk des Marquis de Sade 371; 4.1. Gut oder Böse-ein Rechenexempel? 371; 4.2. Der Pornograph und die Glücksphilosophie des 18. Jahrhunderts 373; 4.3. Das Recht des Stärkeren 375; 5. Aufklärung und Revolution: Die Apologie eines Zeitgenossen 376; Anmerkungen 378; Bibliographie 394.

Anne Christel Recknagel: Diderots Erzählung Les deux amis de Bourbonne. Eine Analyse unter produktions- und rezeptionsästhetischen Gesichtspunkten 401

1. Die Fabel 402; 2. Der historische Entstehungskontext 403; 3. Analyse der Erzählung 404; 4. Die poetologische Reflexion 407; 5. Exkurs: Zur Entwicklung des "conte" im 18. Jahrhundert 410; 6. Die künstlerische Applikation der Poetik - die Deux amis als Einlösung der poetologischen Forderungen 411; 7. Die Moral der Geschichte 414; 8. Zur Vieldeutigkeit Diderotscher Texte 415; Anmerkungen 417. 


\section{Vorwort}

Schon beim ersten Blick in das Inhaltsverzeichnis wird auch der freundlich-kritische Leser rasch feststellen, was fehlt. Die Autoren der Beiträge und die Herausgeber wissen, auf welche Namen, Texte und geschichtlichen Zusammenhänge sie verzichtet haben; sie können sich auch vorstellen, daß ein Kenner der französischen Literaturgeschichte gerne vieles über hochberühmte, weniger berühmte und fast vergessene Dichter, Dramatiker und Romanciers, über offene, verborgene und vermutliche literarische Tendenzen nachschlagen, verifizieren oder falsifizieren möchte. Indessen sind wir uns als Herausgeber mit den Autoren darüber einig gewesen, daß wir weder eine Sammlung von Textinterpretationen noch eine komplette Literaturgeschichte noch gar eine Art literarisches Lexikon anstreben. Mit den vorliegenden Abhandlungen, die monographisch oder problemgeschichtlich orientiert sind, sollen vielmehr einzelne Epochen der französischen Literaturgeschichte exemplarisch veranschaulicht werden. Wir wollen nicht mit umfassenden literaturgeschichtlichen Perioden über vieles hinwegreden; wir schweigen lieber über vieles und sprechen von einigen Schriftstellern, Werken, Gattungen und Problemen ausführlicher. An welche geneigten Leser haben wir nun dabei gedacht?

Nach unseren Erfahrungen fällt es oft schwer, Romanistik-Studenten eine sbrauchbare`Lektüre für den `Einstieg`, für die grundlegende Orientierung und Motivierung, zu empfehlen. Entweder sind die französischen Literaturgeschichten svollständig. und finanziell erschwinglich; dann findet man darin viele Namen, Daten und geistreiche Andeutungen; man muß sich allerdings mit einer äußerst resümierenden Information begnügen, die häufig aus zweiter Hand stammt und die den Uneingeweihten oder den Anfänger daher abstrakt bis unverständlich anmutet. Oder aber die Literaturgeschichten sind vollständig, ausführlich, gründlich, mit Textbeispielen belegt und reich bebildert; dann umfassen sie allerdings so viele Bände und erscheinen so teuer, daß ein Neuling den Mut verliert und das Portemonnaie zurücksteckt. Doch außer dem Anfänger wird auch der Romanist von Beruf neben diesen beiden eben erwähnten Typen von Literaturgeschichten und ihren Varianten die drei vorliegenden Bände fruchtbringend benutzen können - sei es als Selbstbestätigung, sei es als ideologische oder kritische Absprungbasis.

Mit diesen Bänden geben wir jedoch in erster Linie den Schülern und Studenten, die sich einem Leistungskurs oder dem Studienfach Französisch widmen, sowie allen historisch und literaturgeschichtlich interessierten Lesern die Möglichkeit, einige 
literarisch und zugleich geschichtlich bedeutsame Epochen des Nachbarlandes, die man auch im deutschen Sprachraum häufiger beschwört, besser kennenzulernen. Dazu bieten wir ihnen textanalytische, gesellschaftsgeschichtlich reflektierende und literarhistorisch argumentierende Studien an. Wir wünschen uns, daß die detaillierte Argumentation der Beiträge und die extensive Wiedergabe mancher Texte Lehrer der Sekundarstufe sowie Hochschullehrer, die einführende Veranstaltungen planen, dazu anregen, ihren Unterricht auf einzelnen Beiträgen dieser Bände aufzubauen.

Wir sind bei unserer Planung von hochschuldidaktischen Erfahrungen und Vorstellungen ausgegangen. Deswegen haben wir, was die Gestaltung der Beiträge anbelangt, eine Synthese zwischen der detaillierten Textinterpretation - einschließlich ausführlicher Zitate aus weniger leicht zugänglichen Texten - und der Eröffnung literaturgeschichtlicher Perspektiven angestrebt. Die Analyse von Texten und Werken steht im Rahmen des Themas der einzelnen Untersuchung durchaus im Vordergrund. Wir wollen nicht allzu rasch und allzu leicht ein Bedürfnis befriedigen, das nach literaturgeschichtlichen Luftbildern drängt. An der Gewinnung eines Überblicks muß der Leser selbst aktiv mitarbeiten; ersparen wollen wir ihm weder die Mühen und Detailbeobachtungen bei einer Wanderung durch die Landschaft sprich: die Analyse der Mikro- und Makrostrukturen der Texte - noch den lehrreichen Genuß des Fernblicks - sprich: die daraus sich ergebenden Einblicke in geschichtliche und literarische Zusammenhänge. Dem Leser wird es so erleichtert, einige literaturgeschichtliche Probleme am konkreten Beispiel nachzuvollziehen und offene Fragen kraft eigener Lektüre der Werke zu verfolgen. Schließlich hoffen wir auch, daß ihn die eindringlichere Darstellung ausgewählter Sachzusammenhänge anregt und ermutigt, sich die Bereiche, die wir nicht berücksichtigt haben, mit Hilfe der Anmerkungen und Bibliographien selbständig zu erschließen.

Wir haben die Erfahrung gemacht, daß die Bedeutung bestimmter Namen, Daten und Werke der französischen Literaturgeschichte für das Selbstverständnis der Franzosen bei Studierenden häufig nur punktuell und oberflächlich bekannt ist. Das mag sicherlich damit zusammenhängen, daß der Spracherwerb die Priorität im Schulunterricht genießt. Allerdings läßt sich auch beobachten, daß in einem Fremdsprachenstudium, das nicht selten nach dreijährigem Französischunterricht aufgenommen und zugleich vom Berufsbild des Sprachlehrers vorgeprägt wird, der quantitativ und qualitativ ausschweifenden selbständigen Lektüre enge Grenzen gesetzt sind. $\mathrm{Ob}$ hierfür allein diese oder jene gesellschaftliche Institution oder Tendenz verantwortlich zu machen ist, bleibe dahingestellt. Es wäre nun unseres Erachtens wenig angemessen, wenn man den Studienanfänger zuerst einmal mit Fakten, Namen und Ismen berieselte - obwohl das möglicherweise einer gewissen Rezeptionserwartung entgegenkäme. Gewiß übersähe und mißachtete man aber auf diese Weise den Erfahrungsreichtum, den auch die Werke der französischen Literatur in hohem Maße gespeichert haben. Ein literaturgeschichtlicher Unterricht, der im Sog der Vollständigkeit und der bio-bibliographischen Wissensvermittlung steht, wird wohl kaum die Muße lassen, mit den Texten selbst zu begründen, warum es sich heute noch oder heute schon lohnt, Rabelais oder Claude Simon zu lesen, welche besonderen kulturellen Erfahrungen ein deutschsprachiger Leser diesen Zeugnissen entnehmen könnte. 
Selbst das Zeitalter des Massentourismus und des organisierten Schüleraustausches hat die Vermittlung des politischen, sozialen und historischen Kontextes der französischen Literatur nicht überflüssig gemacht. Im Gegenteil - trotz offener Grenzen und Restaurants scheint sich die Wahrnehmung des Fremden, seines kulturellen Kodes häufig wie im Niemandslands zu bewegen. Die Lektüre der mehr oder weniger klassischen Literatur dürfte oft instruktiver sein als eine Busfahrt oder mancher Fernseh-Blick.

Die Autoren haben bei der Darstellung der literaturgeschichtlichen Themen Wert darauf gelegt, den Blick und die Gedanken des Lesers von den Texten auf Probleme und Epochen der französischen Gesellschaft zu lenken. Betrachten wir diese literarischen Texte als Modelle der Wirklichkeit, so haben sie als solche auch einen Abbildcharakter: Als Antworten auf bestimmte historische Prozesse oder auf eine geschichtliche Situation können sie uns verständlicher, wenn nicht plausibler erscheinen. Vor allem wird man ein literarisches Modell als Versuch verstehen dürfen, neue Aspekte seiner zeitgenössischen Wirklichkeit zu beleuchten, ohne daß man es einem absoluten Wahrheitsanspruch unterwerfen dürfte. Denn diese Art von Modellen und die Reichweite ihrer Aussagen werden in nicht unerheblichem Maß von den subjektiven Bedingungen ihres Schöpfers beeinflußt, von Sympathien und Antipathien des Schriftstellers, von seinem mehr oder weniger begrenzten Wissens- und Erfahrungsstand. Außerdem sind die unter dem Kriterium der ästhetischen Wahrnehmung geschaffenen Modelle zwei- und vieldeutiger, sprich: freier als die Modelle, die für die Naturbeherrschung und für das Überleben entworfen und appliziert werden. Mit ästhetischen und in diesem Sinn literarischen Modellen kann wohl kaum unmittelbar Praxis bewältigt werden - selbst wenn Schriftsteller sich darüber Illusionen hingegeben haben und immer wieder hingeben.

Literarische Modelle werden von einzelnen Individuen rezipiert, diese können sie als geistige Anregung, ideologische Bestätigung, Provokation oder als Absurdität empfinden und zum Tagesgeschäft übergehen - Häuser und Flugzeuge bauen, Politik betreiben, Gesetze abfassen, moralische Unterweisungen erlassen. Trotzdem beobachtet man, daß literarische Modelle - selbst die der Vergangenheit - dann und wann Verwirrung stiften, Ärgernis und Empörung erregen, Wirkungen haben. Sie bilden also nicht nur ab oder verschönern, was man ohnehin weiß; dann fände sich ja der Literaturkonsument immer zufrieden in ihnen wieder und mit ihnen ab. Sie können wohl auch über traditionelle Formen des Weltverständnisses hinausweisen, andere Bewertungen vorschlagen, neue Formen und Inhalte der Wahrnehmung eröffnen; es können Modelle der Wirklichkeitserfassung sein, die noch nicht, nicht mehr oder überhaupt niemals applikabel erscheinen. Auf diese Weise brechen sie verkrustete Formen des Weltverständnisses auf - ein Prozeß, den man wahrscheinlich erst aus der historischen Distanz und dann nur umrißartig beschreiben kann.

Wir beabsichtigen nicht, die Erörterungen der Zusammenhänge zwischen Sozialgeschichte und Literaturgeschichte auf der dürren Weide der Methodendiskussion im Kreis herumzuführen. Wir schätzen es auch nicht, wenn unterhaltende, interessante und aufschlußreiche literarische Texte als Belegmaterial für kühne gesamtgesellschaftliche Bogenbauten nur in den Anmerkungen verpackt zu finden sind. Wir 
möchten dem Leser vielmehr Gelegenheit geben, die literarästhetischen, politischen, sozialen, individual- und sozialpsychologischen Implikate der literarischen Modelle einzusehen und ihre Entschlüsselung zumindest an Beispielen zu erproben. Wir meinen, daß viele sogenannte literatursoziologische oder ideologiekritische Fragen dem literarischen Werk selbst zu entnehmen sind.

Bei der Auswahl der Themen haben wir einerseits versucht, den schulischen und universitären Gebrauchswert dieser Bände zu berücksichtigen; deswegen glauben wir, daß eine Schwerpunktbildung im 19. und 20. Jahrhundert durchaus zu rechtfertigen ist. Andererseits haben wir Themen einbezogen, die in einführenden Veranstaltungen oder gestrafften Literaturgeschichten vielfach unterbelichtet bleiben müssen, die aber - wie etwa Moralistik, Trivialliteratur, Schriftsteller und Politik zwischen den Weltkriegen - gerade für die gesellschaftsgeschichtlichen Aspekte der Literaturgeschichte sehr aufschlußreich sind.

Selbstverständlich wird man sich bei einer solchen Themenauswahl immer wieder mit der mehr oder weniger relativen Vorstellung des Repräsentativen auseinandersetzen müssen. Warum beginnen wir zum Beispiel mit Rabelais? Will man die neuere französische Literatur nicht schon mit Villon einsetzen lassen, so wird man wesentliche Aspekte des 16. Jahrhunderts auch aus der Analyse der Pléiade- Dichtung oder der Novellistik oder der Essais von Montaigne gewinnen können. Aber erfährt man aus diesen Beispielen mit der gleichen Anschaulichkeit wie aus Rabelais' Werk, daß die Grenzen des Wissens in allen Bereichen aufbrechen, daß der Humanismus auch volkstümliche Traditionen aufgesogen hat? Die Überlegung, daß in erzählenden Werken die Probleme einer Epoche etwas weniger komplex vermittelt dargestellt werden als etwa in der Lyrik und folglich auch leichter zu erkennen sind, hat insgesamt zu einer gewissen Vorliebe für Romanautoren geführt, die wir aber mit der anvisierten Lesergruppe rechtfertigen zu können meinen. Ein weiterer Gesichtspunkt bei der Auswahl der Beiträge war die Annahme, daß manche Themen und Schriftsteller, wie etwa Stendhal, Balzac, Flaubert und Zola, auf ein gewisses Vorverständnis des deutschen Lesers hoffen können. In diesen Fällen haben wir uns darum bemüht, weniger bekannte Werke in den Vordergrund der Untersuchungen zu rücken.

Wir danken allen unseren Autoren dafür, daß sie die langwierige Planung dieser Bände geduldig ertragen haben; wir danken einigen Autoren dafür, daß sie noch geduldiger als andere die Veröffentlichung abgewartet haben. Wir danken dem Verlag, daß er uns trotz der langen Vorbereitung und der steigenden Druckkosten eine Tür offen gelassen hat.

Berlin und Mannheim im April 1981. 